\title{
Australian Injury Comorbidity Indices (AICls) to predict burden and readmission among hospital-admitted injury patients
}

\author{
Dasamal Tharanga Fernando ${ }^{{ }^{*}}$ D, Janneke Berecki-Gisolf ${ }^{1}$, Stuart Newstead ${ }^{1}$ and Zahid Ansari $^{2}$
}

\begin{abstract}
Background: Existing comorbidity measures predict mortality among general patient populations. Due to the lack of outcome specific and patient-group specific measures, the existing indices are also applied to non-mortality outcomes in injury epidemiology. This study derived indices to capture the association between comorbidity, and burden and readmission outcomes for injury populations.

Methods: Injury-related hospital admissions data from July 2012 to June 2014 (161,334 patients) for the state of Victoria, Australia were analyzed. Various multivariable regression models were run and results used to derive both binary and weighted indices that quantify the association between comorbidities and length of stay (LOS), hospital costs and readmissions. The new and existing indices were validated internally among patient subgroups, and externally using data from the states of New South Wales and Western Australia.

Results: Twenty-four comorbidities were significantly associated with overnight stay, twenty-seven with LOS, twenty-eight with costs, ten with all-cause and eleven with non-planned 30-day readmissions. The number of and types of comorbidities, and their relative impact were different to the associations established with the existing Charlson Comorbidity Index (CCl) and Elixhauser Comorbidity Measure (ECM). The new indices performed equally well to the long-listed ECM and in certain instances outperformed the CCl.

Conclusions: The more parsimonious, up to date, outcome and patient-specific indices presented in this study are better suited for use in present injury epidemiology. Their use can be trialed by hospital administrations in resource allocation models and patient classification models in clinical settings.
\end{abstract}

Keywords: Burden, Comorbidity, Cost, Index, Injury, Readmission

\section{Background}

Hospital-admitted injury patients can experience adverse outcomes during the course of the hospital stay, and comorbidities have the potential to increase that burden. Adverse outcomes include complications, extended hospital stay, readmission to hospital, discharge to longterm nursing care facilities and death. Previous research

\footnotetext{
* Correspondence: tara.fernando@monash.edu

${ }^{1}$ Monash University Accident Research Centre, Monash University, Clayton Campus, 21 Alliance Lane, Clayton 3800, Victoria, Australia

Full list of author information is available at the end of the article
}

shows that among hospitalised patients, burden-related outcomes such as readmission to hospital [1-3], length of stay in hospital (LOS) $[4,5]$ and hospital costs [6-8] are associated with comorbidity. Therefore, comorbidities can increase the likelihood of adverse events occurring, and among injury patients, comorbidities may worsen their outcomes. The ability to quantify the effect of comorbidities for injury patients can assist in predicting outcomes in clinical settings and estimating injury burden in epidemiological research.

(c) The Author(s). 2021 Open Access This article is licensed under a Creative Commons Attribution 4.0 International License, which permits use, sharing, adaptation, distribution and reproduction in any medium or format, as long as you give appropriate credit to the original author(s) and the source, provide a link to the Creative Commons licence, and indicate if changes were made. The images or other third party material in this article are included in the article's Creative Commons licence, unless indicated otherwise in a credit line to the material. If material is not included in the article's Creative Commons licence and your intended use is not permitted by statutory regulation or exceeds the permitted use, you will need to obtain permission directly from the copyright holder. To view a copy of this licence, visit http://creativecommons.org/licenses/by/4.0/ The Creative Commons Public Domain Dedication waiver (http://creativecommons.org/publicdomain/zero/1.0/) applies to the data made available in this article, unless otherwise stated in a credit line to the data. 
Currently there are several established methods for quantifying the association between comorbidities and outcomes. These include capturing the presence of at least one, each or a count of all comorbidities, and the use of comorbidity indices such as the Charlson Comorbidity Index (CCI) [9] and the Elixhauser Comorbidity Measure (ECM) [10]. The CCI allocates a weighted summed score for seventeen comorbid conditions while the ECM is a binary representation of thirty conditions. The CCI was originally derived in 1987 to assess the effect of comorbidity on mortality and is one of the most widely used. It was last updated in 2011 by Quan et al. [11]. The ECM was enhanced by van Walraven et al. in 2009 into a total score [12] and is less popular than the CCI. Some research considered the ECM as inconvenient due to the high number of comorbidities, arguing this may result in over-fitting [13].

Factors associated with outcomes for injury patients are different to those for general hospital-admitted patients $[10,13,14]$. Comorbidity measures should therefore consider the study population as well as the outcome. The type of data available, disease prevalence and clinical relevance also play an important role when deriving such measures. Reflecting this, using existing indices such as the CCI (derived for mortality) does not work well for other burden outcomes $[6,14]$.

The purpose of this study was: (1) to derive and validate new indices to establish the association between comorbidity, and readmission and burden-related outcomes, such as LOS and hospital costs, among hospitaladmitted injury patients using Australian administrative datasets; and (2) to compare the performance of the new indices with the $\mathrm{CCI}$ and $\mathrm{ECM}$.

\section{Methods}

\section{Data sources}

An analysis of existing morbidity data from the states of Victoria (Victorian Admitted Episodes Dataset (VAED)), New South Wales (NSW) (Admitted Patient Data Collection (APDC)) and Western Australia (WA) (Hospital Morbidity Data Collection (HMDC)) was carried out. The Victorian dataset was used for deriving injury comorbidity indices and the interstate datasets were used for external validation. Data provision and linkage were undertaken by the Centre for Victorian Data Linkage (CVDL) in Victoria, the Centre for Health Record Linkage $(\mathrm{CHeReL})$ in NSW and the Data Linkage Branch (DLB) in WA.

These datasets capture all public and private hospital admissions, and contain patient demographics and morbidity information. The morbidity information includes 40 diagnosis fields for Victoria, 51 for NSW and 78 for WA, consisting of disease, injury and external cause data, coded to the ICD Tenth Revision, Australian
Modifications (ICD-10-AM) [15]. Mortality data were extracted from (i) the Registrar of Births, Deaths and Marriages in NSW and (ii) Mortality Register in WA. Direct and indirect hospital costs for Victoria was sourced from the Victorian Cost Data Collection by CVDL and linked to the VAED.

\section{Data linkage}

Data linkage (using patient-specific identifiers) was performed by CVDL using deterministic data linkage for the Victorian data while $\mathrm{CHeReL}$ used probabilistic matching techniques for NSW, and DLB in WA used a multi-faceted process which includes numerous automated and manual sub-processes. CVDL estimates the false positive rate to be between 0.5 to $1 \%$, and the false negative match rates to be between 1 and 2\% [16] and $\mathrm{CHeReL}$ estimates the false positives to be around $0.5 \%$ [17]. It is expected that the false negatives in the Western Australian Data Linkage System exceed the number of false positives, estimates for which are not attempted by the linkage unit [18].

\section{Case selection}

Injury cases were identified and selected as those with records containing an ICD-10-AM diagnosis code in the range "S00" to "T75" or "T79" in the first appearing diagnosis field in the morbidity datasets; a practice commonly used for national reporting [19]. Case selection was further limited to index injury (i.e., the first injury record in the morbidity dataset for a patient during the study period) and residents belonging to each state. Consecutive records of inward transfers from other hospitals or statistical separations within the same hospital were considered to be part of one episode. Children less than 15 years of age were excluded when deriving the indices and validating; the rationale being that children differ to the rest of the cohort in terms of comorbidity prevalence.

\section{The Victorian cohort}

The comorbidity indices derivation cohort consisted of adult patients with an index injury admission between 01 July 2012 and 30 June 2014 (140,094 patients). They were followed up over a period of two years for subsequent hospital admissions.

\section{The NSW and WA cohorts}

The same selection and followup process was used as for the Victorian cohort using the APDC (201,791 patients) and HMDC (71,771 patients). These patients were also followed up in the mortality data to identify patients for censoring; those who died within 30 days of hospital-discharge did not have a possibility for 
readmission and were excluded from analysis pertaining to readmissions within 30 days $[20,21]$.

\section{Coding of outcomes, factors and comorbidities Outcomes}

Two outcomes related to LOS, one cost outcome and two outcomes related to readmissions were chosen for index derivation, some details of which has been published before [22].

The LOS days in the morbidity datasets were re-coded as (1) a binary code "0" for those who were discharged on the same day and " 1 " for those who stayed overnight, and (2) the number of days in hospital as a continuous variable for the index episode for those who stayed at least overnight. Case selection for the second LOS outcome was limited to those who stayed less than 1 month in the index episode ( $92 \%$ of cases).

Hospital costs were recorded in Australian dollars (AUD), and were available for Victoria from July 2012 to June 2015 only for those admitted to public hospitals. Costs were standardized to 2012 dollar values using the Australian Consumer Price Index [23]. Due to its skewness it was log transformed for analysis.

Two forms of readmissions (any-cause and nonplanned) were coded, both with a binary outcome: "1" for patients readmitted within 30 days and "0" otherwise, limited to the first occurring readmission. Patients who died in hospital and those who left against medical advice were excluded.

\section{Factors}

The factors considered were age, sex, body-region, injury type, injury severity, SEIFA (Socio Economic Indexes For Areas), country of birth, geographic region and comorbidity. The coding is similar to that used in our previous studies (Fernando et.al. 2019 \& Fernando et al., 2020) [22, 24]. Injury severity was assessed using the ICD-based Injury Severity Score (ICISS) [25]. Using the worst injury method [26], a serious injury was considered to be one with an ICISS less than or equal to 0.941 (survival probability of $94.1 \%$ ) [27]. The survival risk ratios used in calculating the ICISS were provided by the National Injury Surveillance Unit [28].

\section{Comorbidities}

A combination of the CCI [9] and ECM [10] comorbidity groups per Quan et al. (2011) [29] and Sundararajan et al. [30] were used to select the list of comorbidities for study. Further details can be found in Fernando et al. (2019 \& 2020) [22, 24].

\section{Statistical analysis}

A negative binomial regression model for LOS, a linear regression model for log transformed costs and a logistic regression model for the binary outcomes (overnight stay, all-cause- and non-planned 30-day readmission) were fitted using multivariable regression. Sociodemographic variables and injury characteristics were entered in the baseline models.

Predictive power of the logistic regression models was assessed using discrimination (area under the receiver operating characteristic curve) and classification tables. The area under the curve (AUC) ranges from 0 to 1 , with a value of under 0.7 representing poor discrimination and anything above that as good discrimination. Classification tables were derived on the basis of using a classification cut-off probability that maximized the combined sensitivity and specificity of the table based on the receiver operating characteristic curve. The adjusted $R^{2}$ and McFadden's $R^{2}$ was used to determine predictive ability for the linear and negative binomial models.

After running the baseline models, comorbidity was added in various forms. Models were compared using the Akaike Information Criterion (AIC) [31]. A difference of less than 10 between two AICs indicates that the model with the additional factors provides no further improvement to the model fit. Using a backward elimination process starting with all thirty-one conditions in the model fitted as binary variables, a final model was derived which excludes comorbidities that no longer improve the model and were hence eliminated by the process.

The significance of interaction terms was tested using the AIC statistic (for model fit) and the improvement to the predictive power of models using the changes in (i) the AUC statistics for logistic models and (ii) adjusted $R^{2}$ and McFadden's $R^{2}$ for the linear and negative binomial models. If both the AIC statistics change and the improvement to predictive powers were significant, then the interaction terms were retained.

The final model containing the binary representation of comorbidities significant to the outcome was retained. This model was given the generic name the "Australian Injury Comorbidity Index". However, depending on the outcome of interest, an extension term describing the outcome is added to the generic name.

A weighted comorbidity index was derived using the final binary model, weights were computed for each comorbid condition using the following: resulting odds ratios (ORs) for each condition from logistic regression, incident rate ratios (IRRs) for the negative binomial regression; and the exponential of the beta coefficients for the linear regression model. The following rules were applied in allocating weights: the condition was dropped from the index if the weight $<1.2 ; 1.2 \leq$ weight $<1.5$ resulted in a score of $1 ; 1.5 \leq$ weight $<2.5=2 ; 2.5 \leq$ weight $<3.5=3$ and so on. These weights were summed up to create the summed weighted score. 
Two more indices were also derived: one parsimonious binary injury comorbidity index for burden and one for readmissions. The first was created using only conditions that were associated with LOS and cost, while the second was based on conditions associated with readmissions.

Finally, a validation of the newly derived indices was carried out. The indices were internally validated on patient subgroups and externally on NSW and WA datasets. For the internal validations, patient subgroups were selected based on demographics and/or injury characteristics. The groups were children $(<15$ years), adults ( $>=65$ years), males, females, adults with non-severe injuries, adults with intracranial injuries, adults with blunt trauma, adults with penetrating injuries and hip fracture patients aged 45 years and over. The validation models were the same baseline model identified for each outcome in Victoria with the addition of the binary index, the weighted index, the burden index, the readmission index, CCI, updated CCI and ECM to represent comorbidity. The models with various indices were assessed for predictive ability using the AUC and classification tables and adjusted $R^{2}$ s. Stata 14.0 (StataCorp) was used to analyze the data [32].

\section{Results}

\section{Overview of the Victorian study population}

Nearly a third $(30.9 \%)$ of the population were 65 years and above, $55.3 \%$ were males and approximately $13 \%$ had serious injuries. More than half (59.6\%) of the patients had a main injury to the extremities and the highest proportion of injuries were fractures (41.3\%) (Table 1).

The median LOS was 1 day (IQR $1-3$ ) for the entire cohort while those with at least one comorbidity had a higher median LOS of 4 days (IQR 1-17). Over twothirds required an overnight stay (68.4\%). The mean hospital cost was AUD 7457.3 (95\% CI 7370.1 - 7544.5) (based on 123,207 episodes of care with complete cost data), while the mean cost for those with at least one comorbidity was nearly double (AUD 14,157.5 (95\% CI 13, $876.8-14,438.2)$ ). Excluding patients who died or left against medical advice, $11.4 \%$ had at least one any-cause and $7.6 \%$ one non-planned readmission to a hospital within 30 days of being discharged for the index admission. These readmissions increased to 18 and $12.7 \%$ respectively for those with at least one comorbidity. Overall, comorbidity increased with age, was higher among females, and higher among patients with seriousinjuries. An overview of the NSW and WA study populations is also presented in Table 1, and details of all three populations in Appendix Table A1.

\section{Multivariable regression modelling} Comorbidity in the Victorian study population (adults)

Alcohol dependence, cardiac arrhythmia, dementia, depression, diabetes, hypertension without complications and renal disease were the most commonly recorded comorbidities among injured adults ( $>15$ year of age) (Table 2). After adjusting for baseline variables such as demographics, and injury characteristics including injury severity, the comorbidities associated with a high burden on the system (LOS and costs) were not the same comorbidities that were associated with high readmission rates.

\section{Baseline models}

Results for the baseline models are presented in Table 3 (model $i$ ). The baseline factors differ for each outcome (see stepwise breakdown of factor inclusion in Appendix Table A2). Age, sex and injury characteristics all improved the model fit for all baseline models except for all-cause readmissions, where adding sex and injury severity did not improve the model fit any further. However, sex was retained in all baseline models for consistency.

Patients from regional areas were more likely to stay overnight compared to patients from metropolitan areas. The baseline model for costs was similar to overnight stay which is expected given costs are highly correlated to LOS. Region of residence was associated with readmissions.

\section{Fitting comorbidity using various measures}

Models $i i$-vii are baseline models with comorbidity fitted using the newly derived binary (ii) and weighted comorbidity indices (iii), existing indices (CCI (iv), updated CCI (v) and ECM (vi)) and the parsimonious indices (conditions common to burden/readmission outcomes alone) (vii) (Table 3). Results from fitting comorbidity using other forms are also presented in an appendix (Appendix Table A2). The model coefficients and weights for each comorbidity, by outcome are presented in Table 4. ROC curves, prediction plots and residual plots of the various models are presented in Appendices A1.1, A1.2, A1.3, A1.4, A1.5.

\section{Interaction effects}

Interaction effects between age and sex, comorbidities and age, and comorbidities and sex, were also modelled. The age-sex interaction was selected because of their known interactions with disease [33], while the age and sex with comorbidity interactions were selected due to the expectation that the severity and impact of comorbidity can vary with age and sex. The interaction terms improved model fit but provided very little or no improvement to the predictive ability of the models, and 


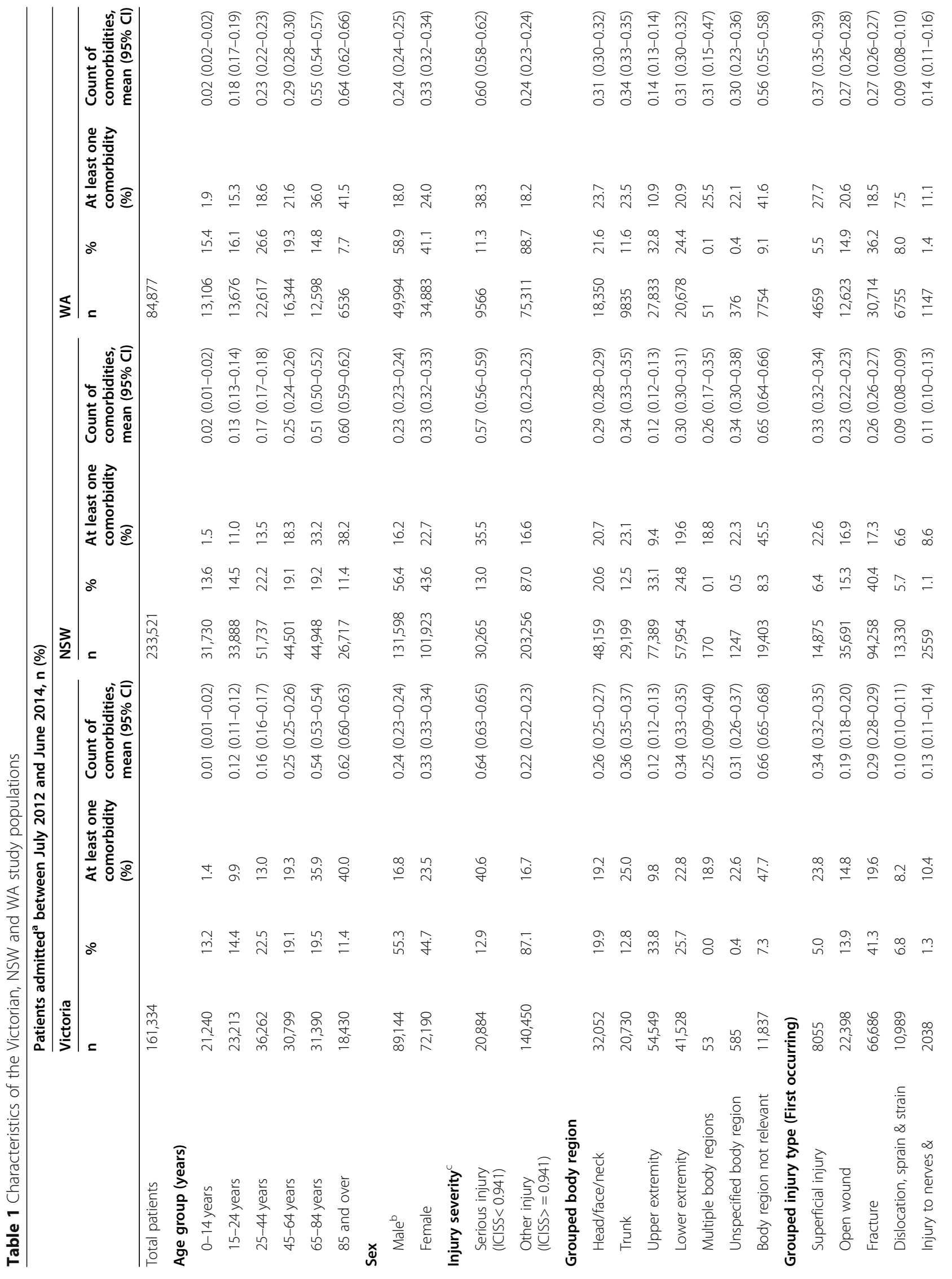




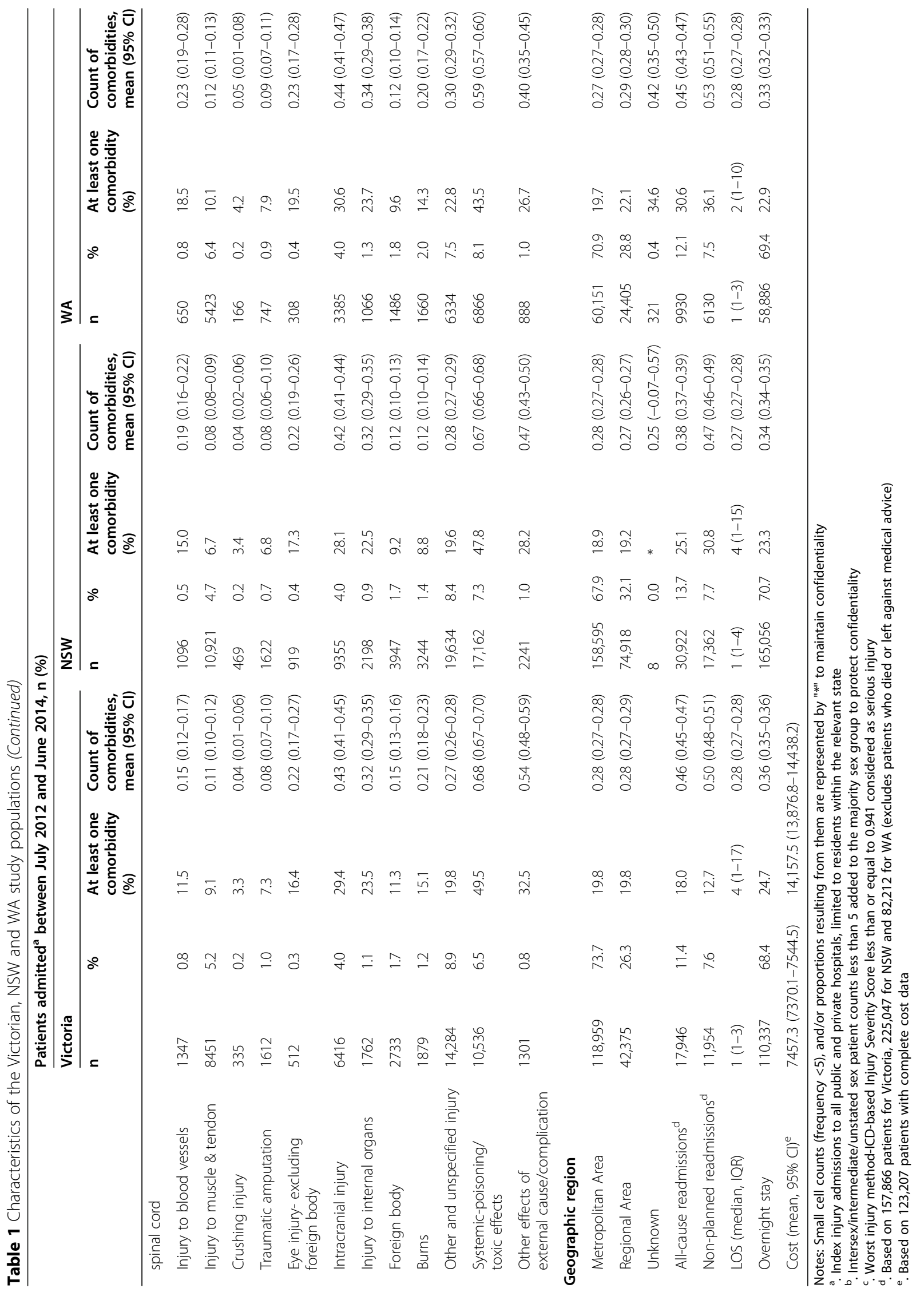




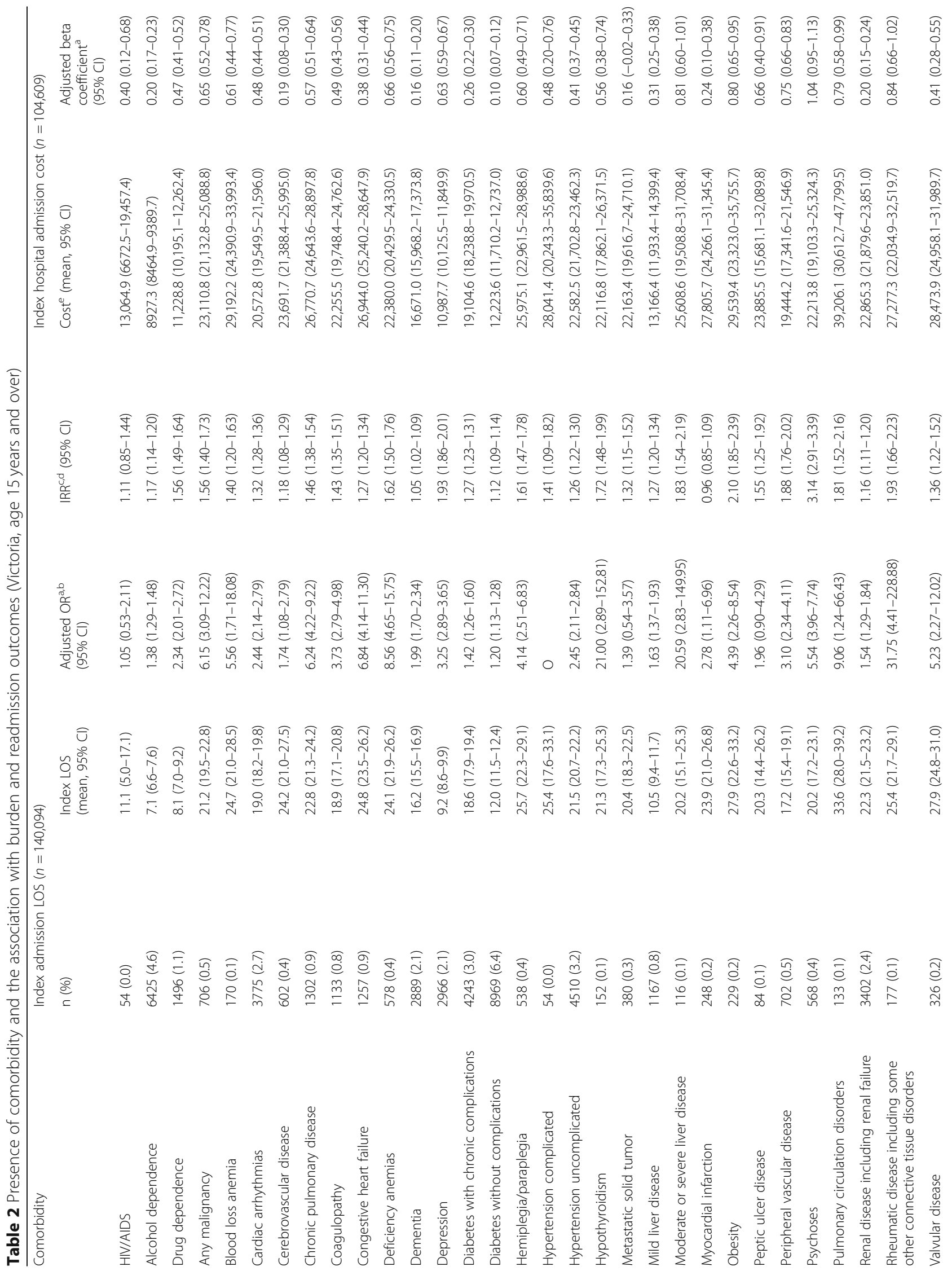




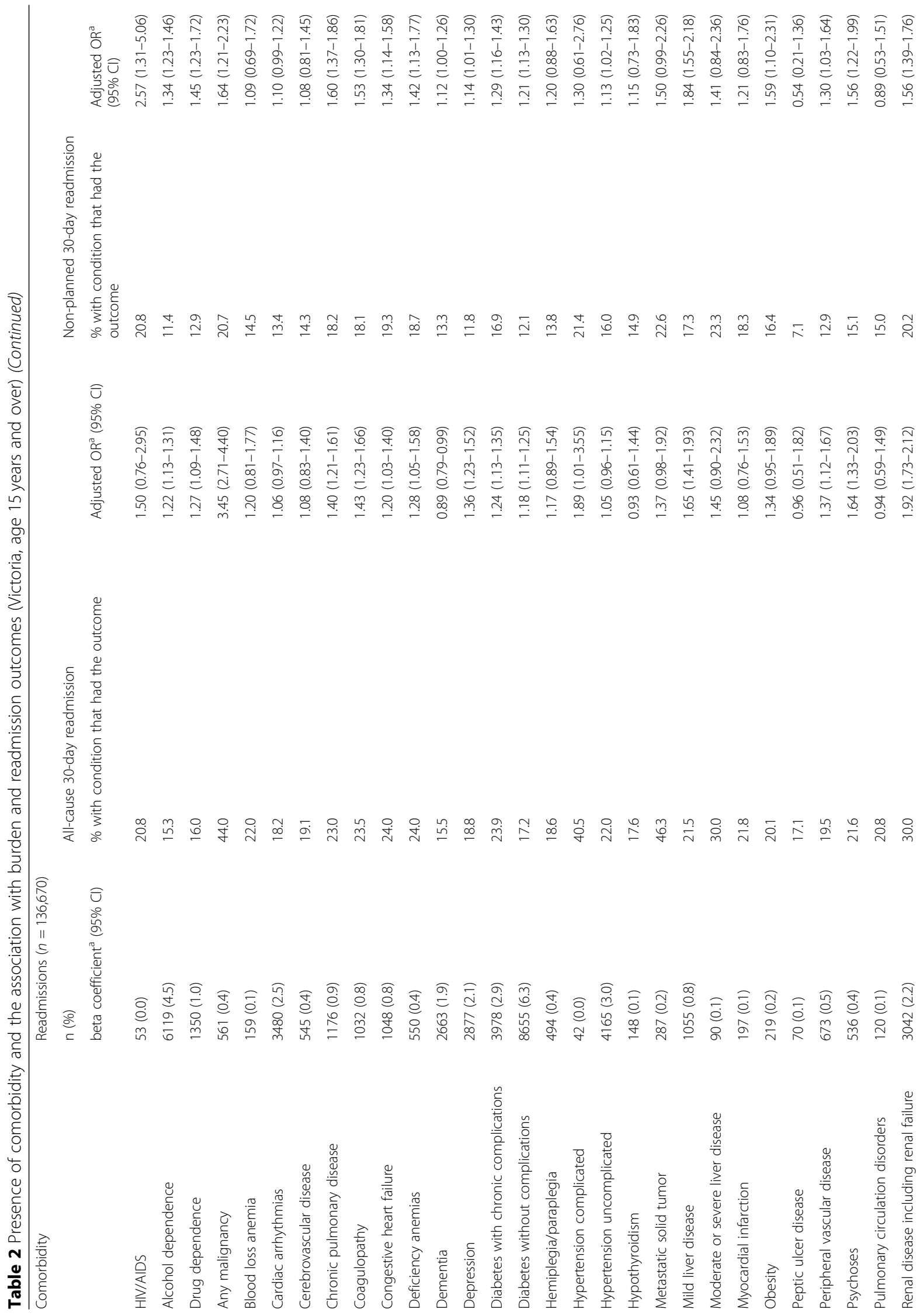




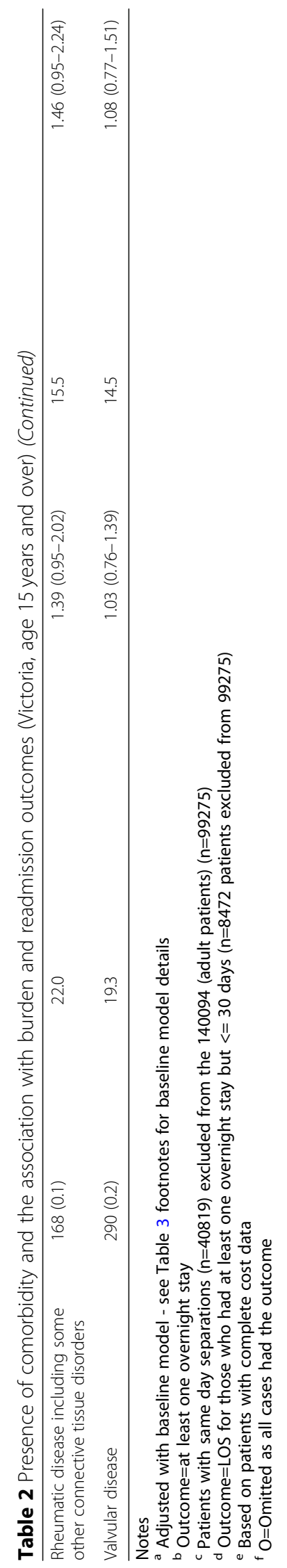




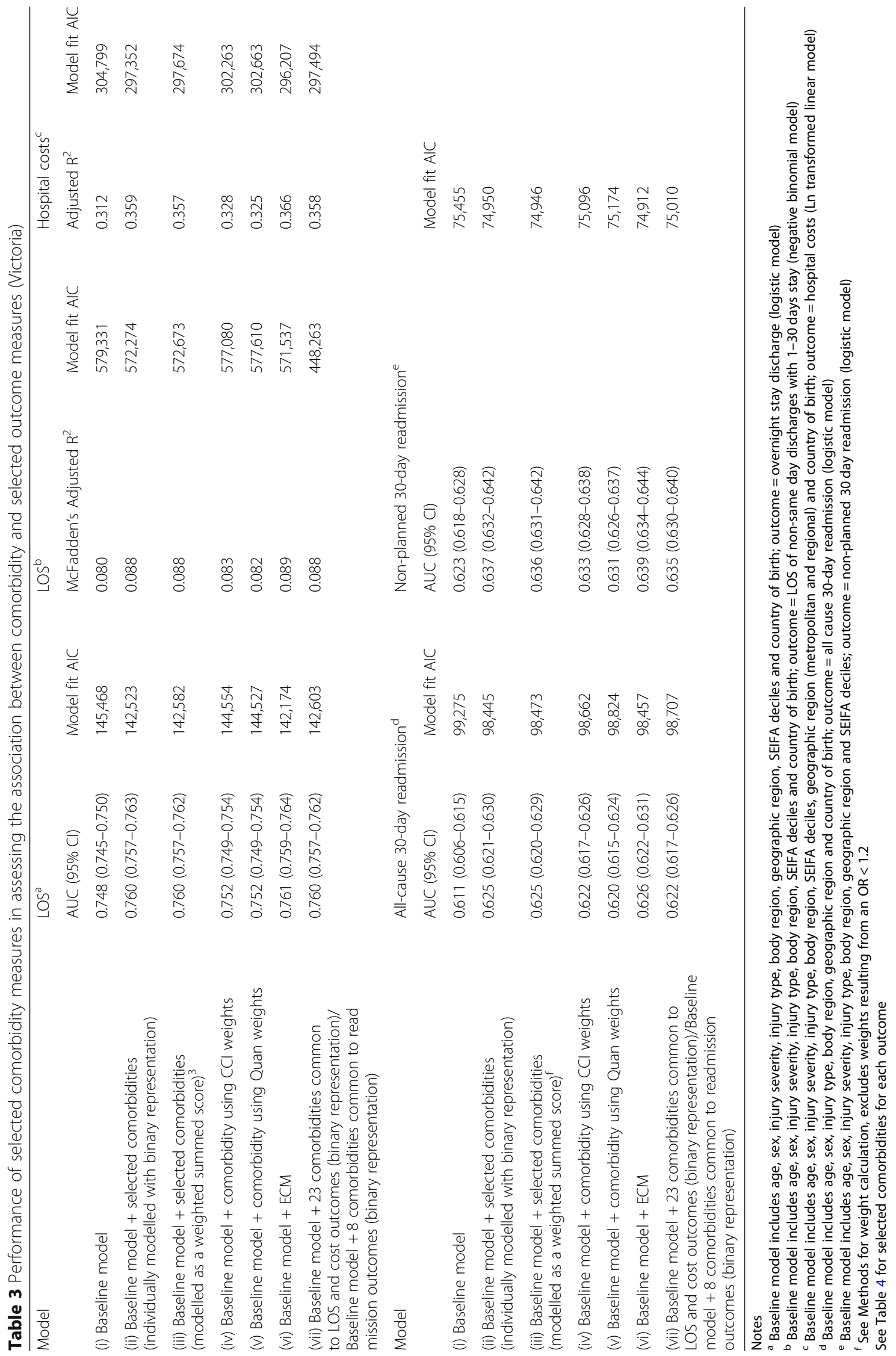




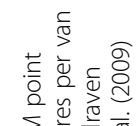

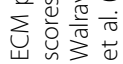

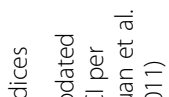

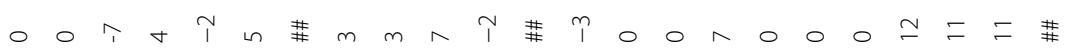

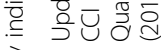

충

흘

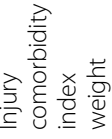

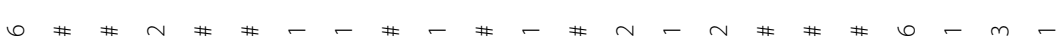

咅

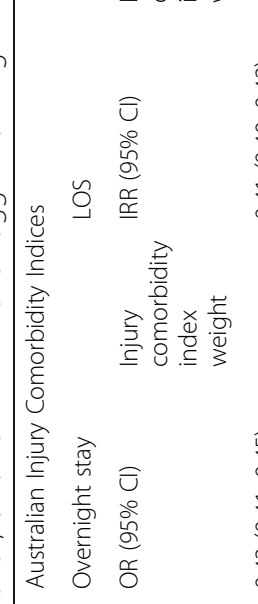

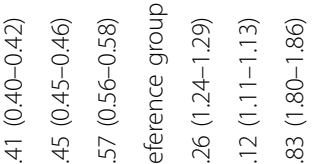

娄 舌

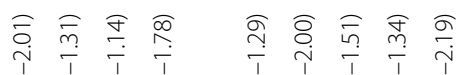

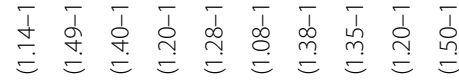

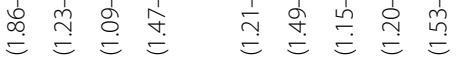

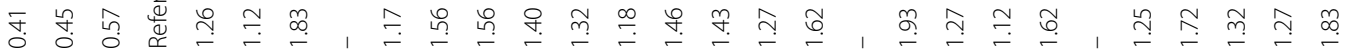

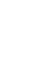
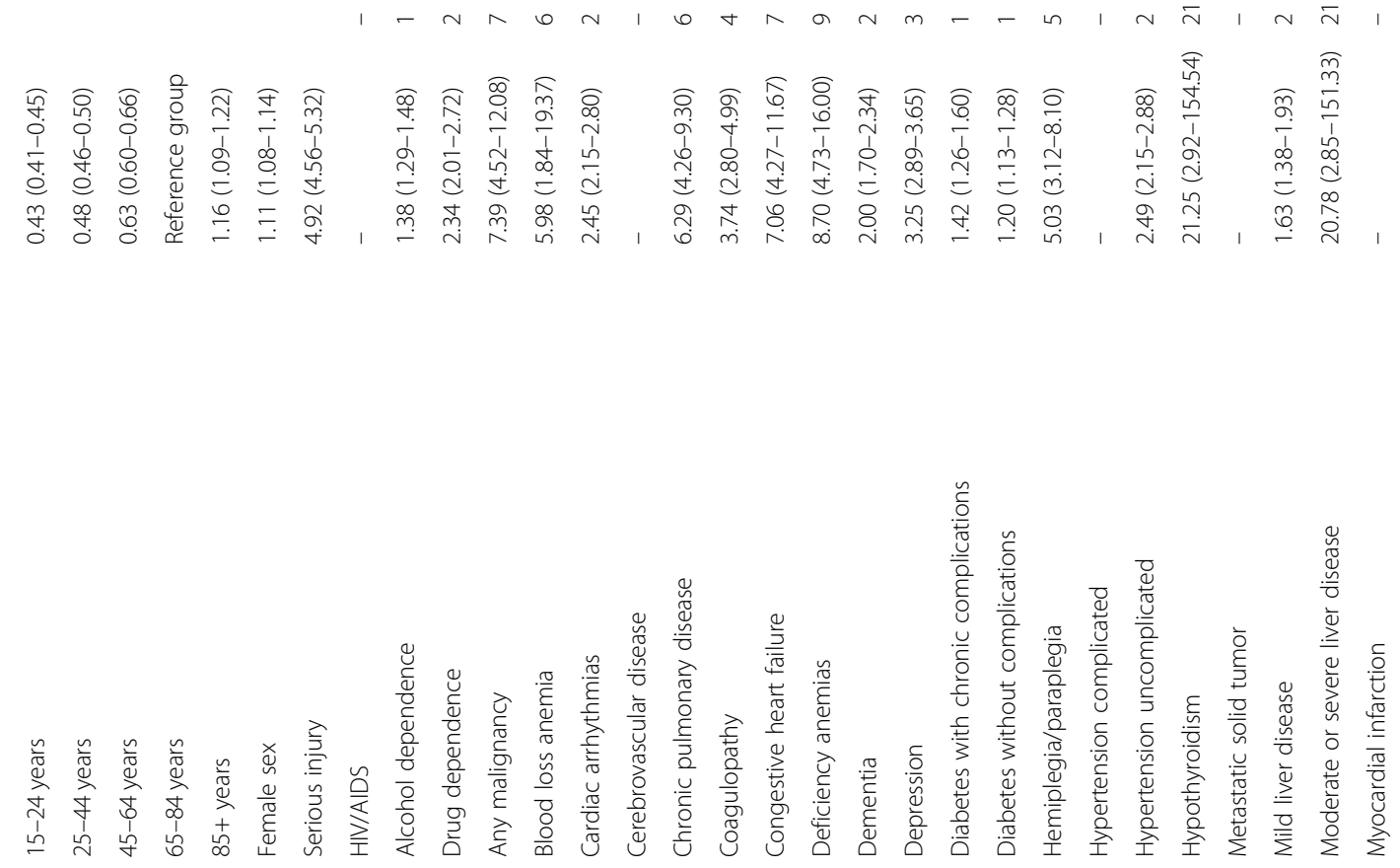


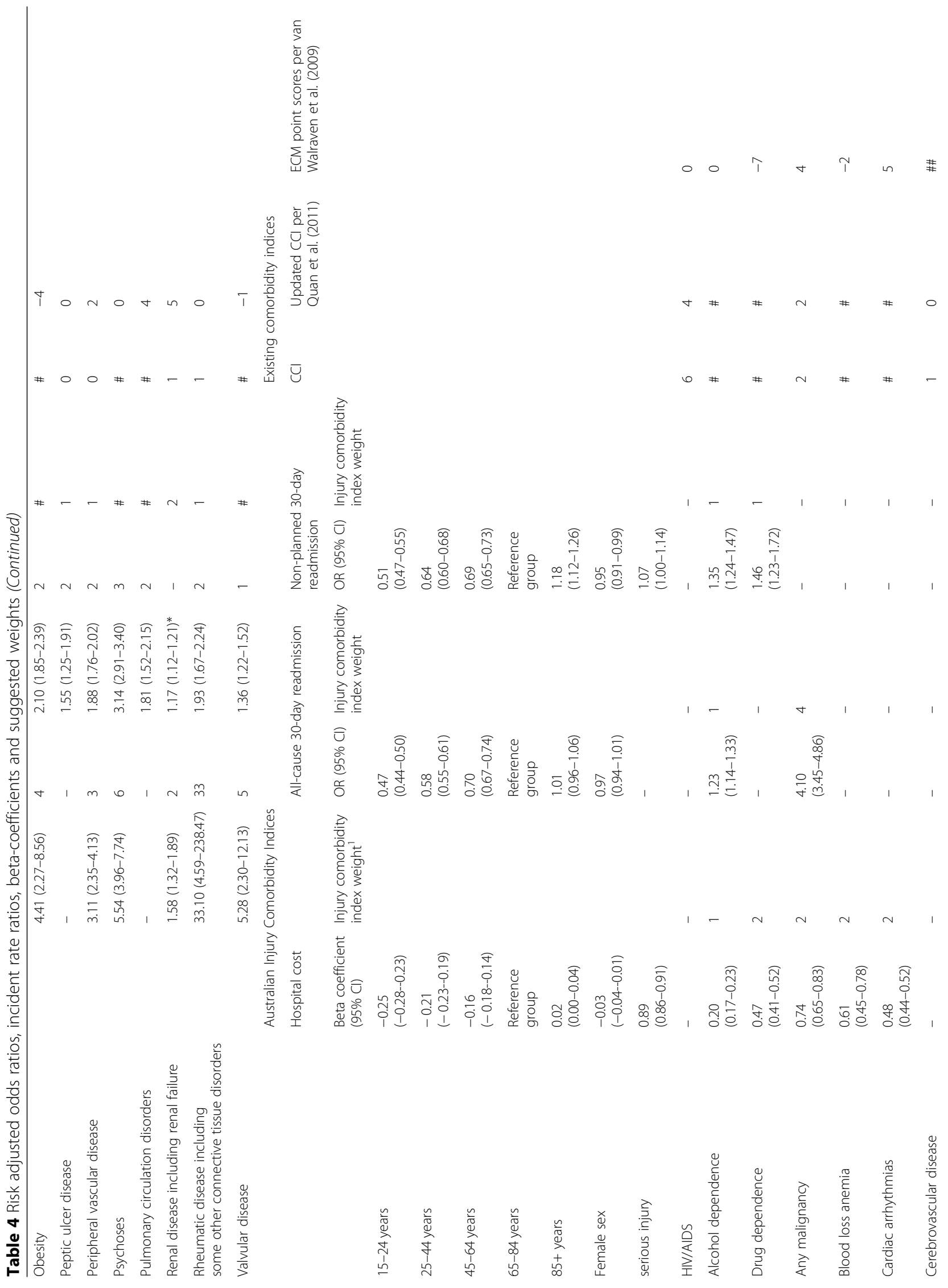




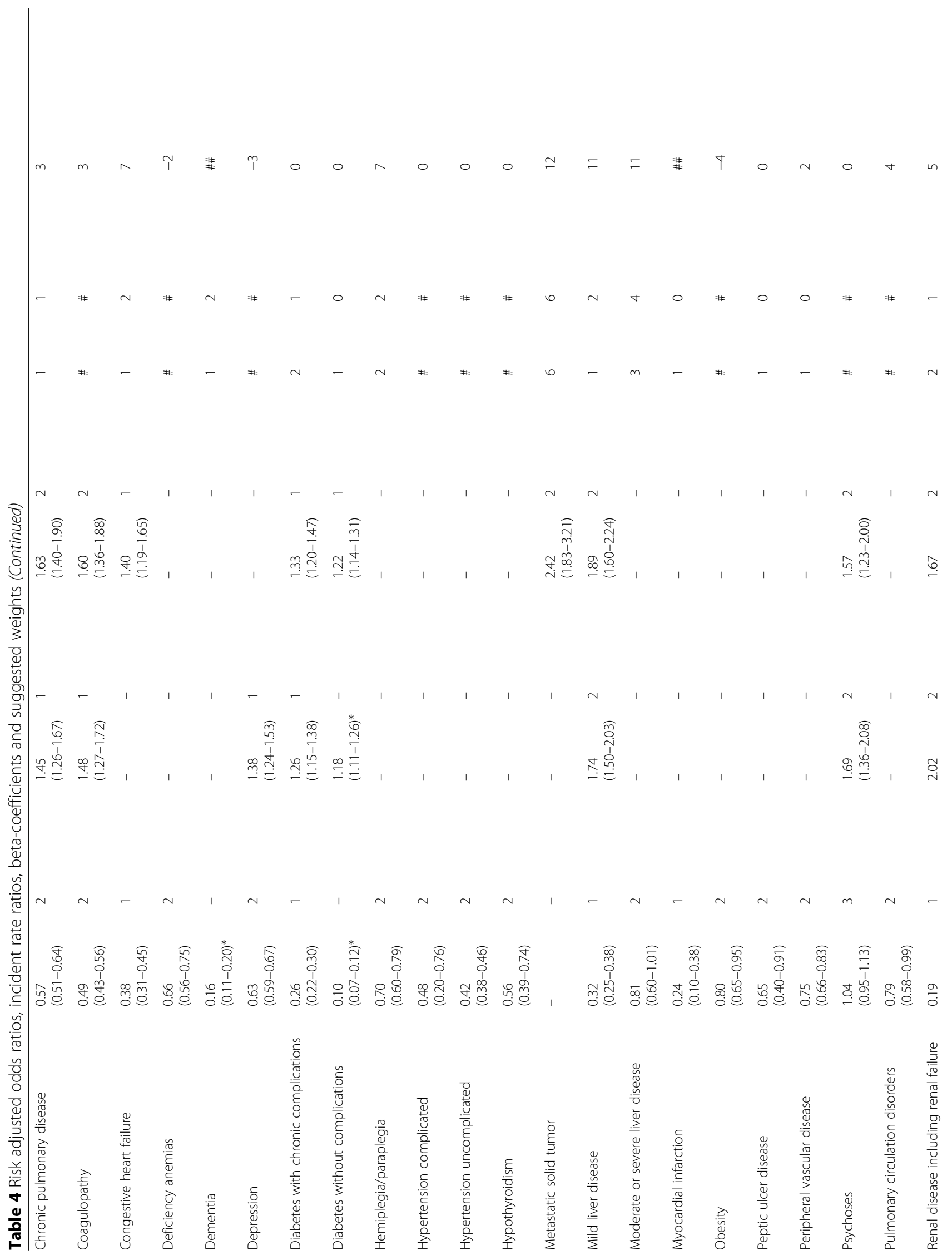




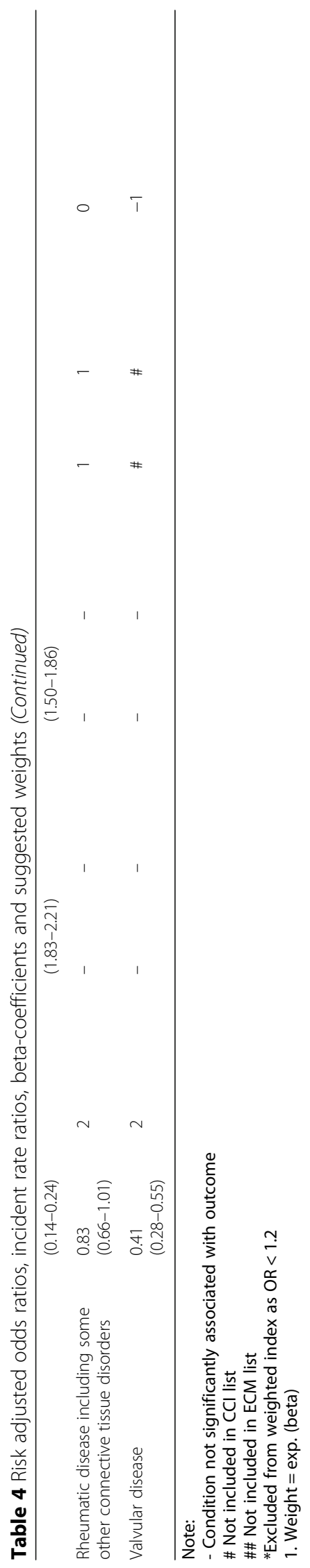




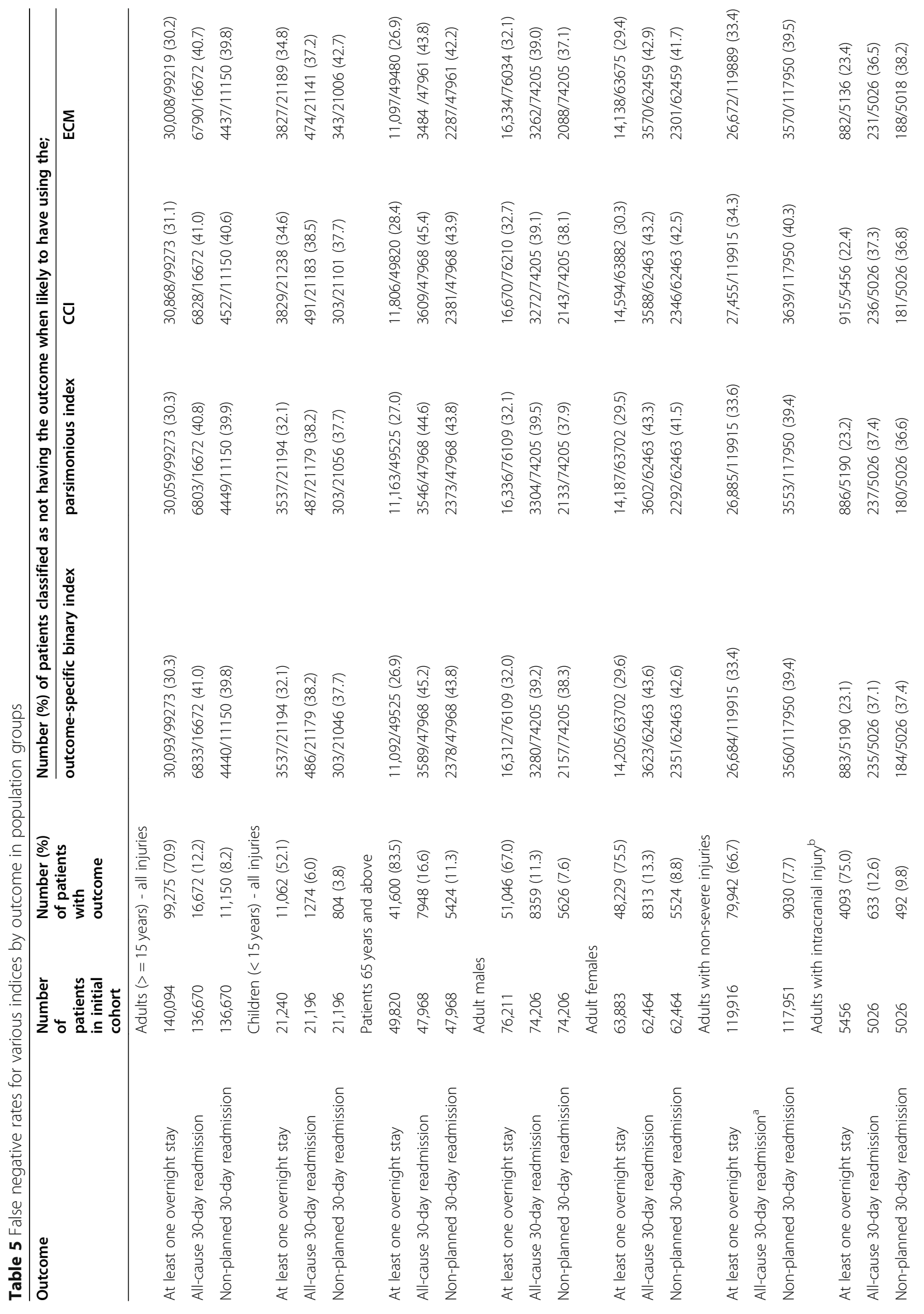




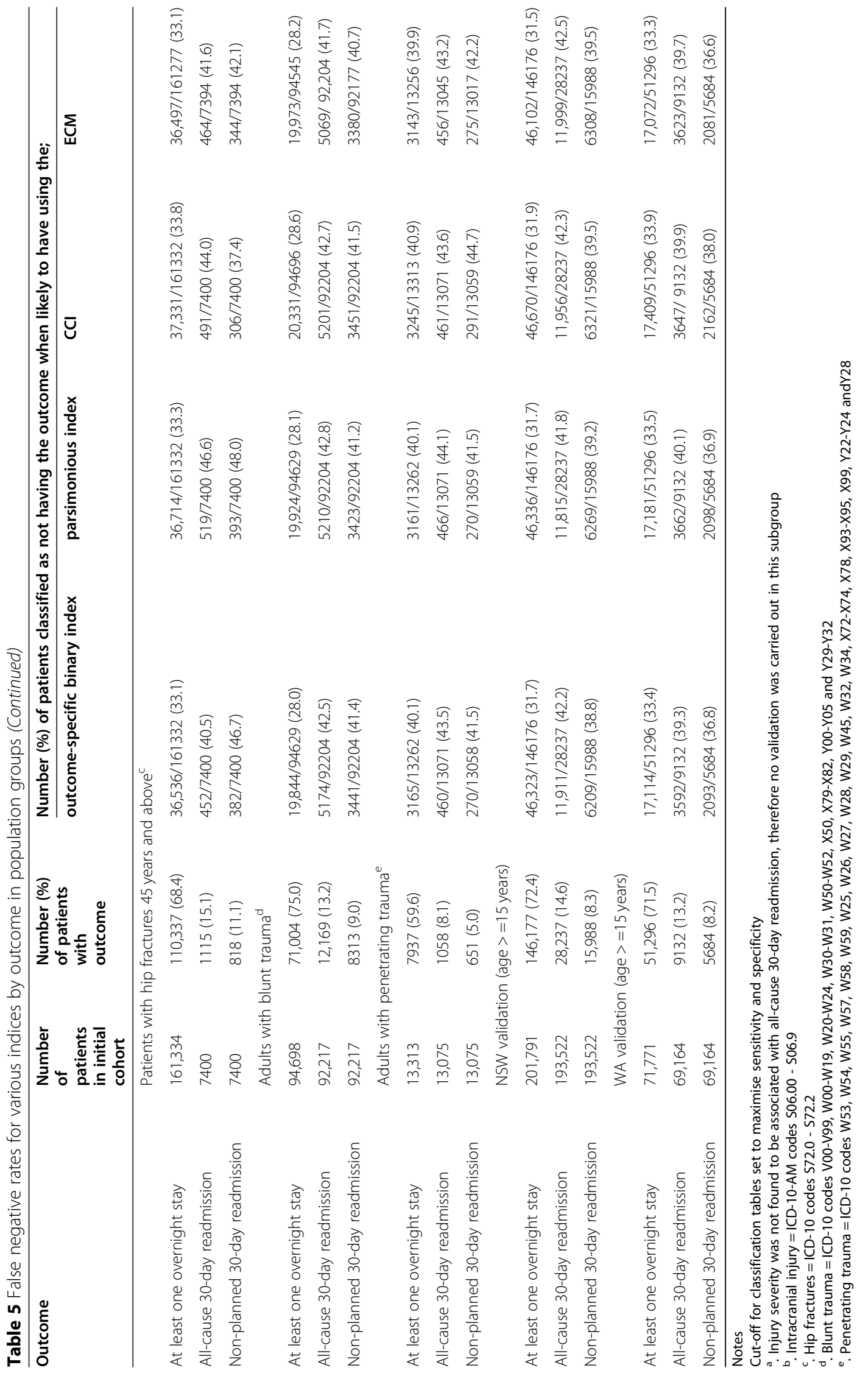


were therefore dropped from the analysis. Plots representing interaction effects are included in Appendix A2.

\section{Overnight stay}

The Australian Injury Comorbidity Index for overnight stay (AICI-os) with 24 comorbidities performed better than the CCI, performed equally to the ECM with the added advantage that it uses fewer comorbidities than the ECM.

The best fit model, as indicated by the lowest AICs; was model $v i$ (containing the ECM), followed by model ii (containing the AICI-os). Models containing the CCI (iv) and updated CCI (v) had much poorer fit. Comparing predictive abilities using the AUC and false negative rates (Table 5), the AICI-os and the ECM performed best; a false negative rate in this instance is when a patient with overnight stay is incorrectly classified as discharged on the same day. Details of model performance can be found in Appendix A3. Discrimination analysis results can be found in Appendix Table A3.

\section{LOS (overnight stay patients)}

Based on model fit, the new index (the Australian Injury Comorbidity Index for LOS (AICI-los)) fits better than the CCI. The AICI-los does not fit as well as the ECM, the only trade-off lies in modelling thirty conditions in the ECM as opposed to only 27 in the AICI-los. In terms of predictive abilities, the differences between models were relatively small. Details of model performance can be found in Appendix A3.

\section{Cost}

Similar to the AICI-los, the Australian Injury Comorbidity Index for costs (AICI-cost) with 28 comorbidities fits better than the CCI and less so than the ECM. Once again, the trade-off between the AICI-cost and the ECM is the number of conditions.

The best fit was once again seen in models with the ECM, followed by the AICI-cost while the CCI and updated CCI had a poorer fit. Predictive power in terms of the adjusted $\mathrm{R}^{2}$ was best in the model with the ECM (36.6\%), followed by AICI-cost (35.9\%), while predictive powers of the CCI (32.8\%) and updated CCI (32.5\%) were lower.

\section{All-cause 30-day readmission}

The Australian Injury Comorbidity Index for all-cause 30-day readmission (AICI-acr) with 10 comorbidities exhibits similar capacity in terms of model fit and predictive power as the existing indices except that it includes fewer conditions than the CCI and ECM.

In terms of model fit, the best were models with the ECM, followed by the AICI-acr, while once again the CCI and updated CCI had a poorer fit. The AUC for all models for this outcome was close to but less than 0.7, indicating that the power of the models was somewhat poor and could probably be improved by factors not already adjusted for. Further details can be found in Appendix A3.

\section{Non-planned 30-day readmissions}

The best fit was again observed for models with the ECM, followed by the Australian Injury Comorbidity Index for non-planed 30-day readmissions (AICI-npr) with 11 comorbidities and poorer fit with the $\mathrm{CCI}$ and updated CCI. There were no significant differences between all the models in terms of AUC statistics and false negative rates. Similar to all-cause readmissions, the AICI-npr has the advantage of having fewer conditions than the CCI and ECM.

\section{Parsimonious indices}

A binary index (model vii) (Table 3) with 23 conditions common in the LOS and cost indices (Australian Injury Comorbidity Index for burden (AICI-b)) and one with eight conditions common to readmissions (Australian Injury Comorbidity Index for readmissions (AICI-r)) was also derived (see Appendix Table A4 for conditions included). Overall, comparing the AUCs, false negative rates and $R^{2}$ s showed that the two parsimonious indices (AICI-b and AICI-r) can be used for predicting the corresponding outcomes without much loss in predictive capacities (Table 3 and Table 5).

\section{Comparison of conditions included in new and existing indices}

Table 4 shows the corresponding weights for each comorbidity by outcome for the AICIs, CCI, Quan update to $\mathrm{CCI}$ and van Walraven update to ECM weights. Conditions like HIV/AIDS, cerebrovascular disease, dementia, metastatic solid tumors, myocardial infarction and pulmonary circulation disorders are allocated weights in the existing indices, while for some of the outcomes they show no association in the new indices.

\section{Internal and external validations}

The newly derived indices along with the CCI and ECM were validated in subgroups of the Victorian population. A detailed discussion of results can be found in Appendix A4 while model results are presented in Appendix Table A5 and false negative rates in Table 5. The new indices and the ECM validated better than the CCI in general across most subgroups for burden outcomes. For readmission outcomes, there was no difference in predictive powers across new and existing indices, the only difference being that the ECM had significantly lower false negative rates among older adults, females and blunt trauma patients. 
External validations were carried out on the entire adult portion of the interstate cohorts. Comorbidity prevalence for these are presented in Appendix Table A6. An explanation of the validation analysis can be found in Appendix A4 with model results in Appendix Table A7 and false negative rates in Table 5. For overnight stay, all indices validated well; NSW a little better than WA and the new indices and ECM better than the $\mathrm{CCI}$, but no significant differences in false negative rates. For LOS, WA had higher $\mathrm{R}^{2} \mathrm{~s}$ than Victoria and NSW, and the ECM performed best followed by the AICI and the CCI. For readmissions, AUCs were low (poor predictive power) for all indices and not significantly different, nor were the false negative rates. WA data overall had better predictive power than the other two states.

Suggestions on how the indices can be used are provided in Appendix A5. A summary table of conditions included in the AICIs, CCI and ECM are presented in Appendix Table A4.

\section{Discussion}

\section{Main findings}

The number and type of comorbidities associated with outcomes vary based on the outcome. The AICIs provide up-to-date, injury and outcome specific parsimonious indices that perform equally well to the long-listed ECM, and in certain instances outperform the widely used CCI.

\section{Study strengths}

This study shows that pre-existing comorbidities associated with burden and readmission outcomes for injury patients are different for each outcome, enforcing the need for outcome-specific indices. For instance, conditions associated with LOS and costs were somewhat similar (though not identical) while conditions associated with readmissions were similar but far fewer in number than burden outcomes. The CCI and ECM has been cited extensively (CCI 29,383 citations and ECM 5450 citations according to Google Scholar as of October 2019) but are often applied to injury populations and non-mortality outcomes when they were originally derived for predicting mortality [34].

The fact that the association between comorbidities and outcomes vary and that the CCI performed better for predicting mortality than LOS and readmissions has been shown in other studies [14, 35]. Both a previous study (Fernando et al., 2020) [24] and the present study gave the same findings for the CCI, ECM and the AICIs.

The new indices allow a more appropriate quantification of the effect of each comorbidity on the outcomes as opposed to using existing indices that are based on older data. More specifically; conditions like HIV/AIDS and metastasis contain the highest risk scores according to the CCI, but the results from this study shows that these conditions have much smaller or no effect on burden and readmission outcomes. Myocardial infarction allocates a certain element of risk in the CCI, but according to the findings here, it only impacted the prediction of costs. Peptic ulcer disease is also allocated a risk element in the $\mathrm{CCI}$ but shows association only with LOS and cost and has no association with readmissions in the present study.

A study by Moor et al. (2008) further showed that the CCI weights assigned to conditions did not correspond to their study coefficients when assessing mortality for trauma patients [36] which the present study also confirmed. Moor et al. (2008) recommended three steps to creating appropriate empirical weights; 1 ) use a large representative database, 2) use appropriate generalizable trauma populations and 3) be up-to-date, all of which the present study has encompassed.

A number of studies in the past have indicated the need for study-specific comorbidity indices or weights $[35,37-40]$. This study has shown the validity of those recommendations by deriving new indices and comparing their performance and parsimoniousness against the existing general indices. The characteristics of the populations, hospital facilities and comorbidity prevalence drive the outcomes.

The only study that derived a comorbidity index for injury populations was by Thompson et al. (2010) [41] which derived the Mortality Risk Score for Trauma using six comorbidities. The predictive power of their index was identical to the CCI and did not show any improvement whereas the indices derived in this study performed a little better.

Another strength of this study is the extensive validations of the new and existing indices carried out in subgroups and external populations. It was seen that in most instances the ECM performed best followed by the AICIs and CCI in predicting outcomes. However, the trade-off on the number of comorbidities modelled and the relevance to the outcome could be the deciding point for whether the ECM or AICI is used.

The new indices may be more versatile for use than the Multipurpose Australian Comorbidity Scoring System (MACSS) which was derived by Holman et al. (2005) [35]. The MACSS was not considered for evaluation in this study because it includes: (i) 102 comorbidities (far less parsimonious than all other indices), (ii) conditions like tuberculosis and (iii) certain symptoms and late effects which are not chronic diseases.

Moor et.al. (2008) [36] and Toson et al. (2015) [14] both claimed that a binary representation of comorbidities was sufficient for establishing the association between comorbidities and outcomes for injury patients and this study has validated that claim. Farley et al. 
(2005) [6] found that costs were better predicted by a count of comorbidities over the CCI; this study found that the binary representation had more predictive power than the count of comorbidities. The difference could be due to the fact that they used diagnosis clusters which we have not investigated in this study.

\section{Study limitations \\ Data limitations}

The $<0.7$ AUC statistics for assessing readmissions indicate that additional variables are required to explain these outcomes. Readmissions could be due to injuries, comorbidities, complications and health service delivery. Adjusting for the cause of readmission, which was not available in these datasets, may help improve the baseline model.

LOS and costs could also be largely driven by hospital facilities as well as the medical and surgical procedures carried out. The distance from a patient's residence to the hospital could be another important factor, all of which was not part of the data included in this study. LOS can also be dependent on the total patient turnout at a hospital; hospitals with low resources may tend to discharge patients sooner resulting in lower LOS, likewise they could result in higher numbers of readmissions. Accounting for these in the baseline models may improve predictive abilities, but not necessarily the comorbidity indices.

\section{Comorbidity capture}

The capture of comorbidities in this study compare well with a previous study of injury patients that used administrative data in Australia. The proportion of injury patients with comorbidities in this study (for Victoria) was 19.5\% while previous research for New South Wales, Queensland and South Australia [42] found 15.6\%; the difference is likely to be attributable to the fact that this study included 31 conditions as opposed to the $17 \mathrm{CCI}$ conditions used in the other study. However, these proportions could be low compared to other studies as the datasets used are administrative data which are not clinically rich as other registries to capture comorbidity.

Drawback of using administrative data The administrative data used in this study does not capture the severity of comorbidities. It may also not capture all comorbidities as the function of most administrative datasets is for informing hospital reimbursement, therefore commodities that are not actively treated within the episode may not be indicated.

Lookback periods The inclusion of lookback periods could improve comorbidity capture. However, the new indices are meant for use at the point of hospital admission, and data for lookback periods are generally unavailable at that point; therefore, inclusion of these will be useful only during research and not in clinical settings.

\section{Practical application}

Care should be taken when using the new indices in populations where the injury profile or the prevalence of comorbidity are different, as was seen in the subgroup analysis (Appendix A4). Further, they should probably not be used to compare the performance of specific hospitals as the driving factors of hospital performance likely vary largely on type, size and location of hospitals.

\section{Case selection bias}

Cases selected for 30-day readmissions analysis in the NSW and WA datasets excludes those that died within 30 days. The exclusion of these patients facing a 'competing risk' for readmission could incorporate some selection bias as it excludes some serious patients who may have the possibility of a readmission within 30 days. However, this proportion is generally around $1 \%$. This exclusion was not carried out for Victoria as the hospital data in this instance was not linked with mortality data.

\section{Implications}

Epidemiological research and resource use predictions for hospital admitted injury patients will benefit from using the AICIs that have been specifically derived and validated for this group of patients. For example, they can be used by hospitals for planning beds, and by health service administrators when budgeting for future hospital expenditure for injury patients with comorbidities.

Another advantage of such indices is that they are less resource intensive given they use information available at point of hospital admission. Clinicians can estimate LOS and the possibility of readmissions for injury patients, adjusting for the effect of comorbidities, and plan the services required accordingly. These indices do not replace clinical knowledge when deciding hospital logistics required for treating patients but maybe used in assisting with the decision-making processes.

\section{Future research}

The AICIs can be further validated in other countries to understand if additional adjustments are required to make them more robust. Another step forward for these indices will be to incorporate comorbidity severity measures, which may improve the indices' abilities.

\section{Conclusion}

Comorbidities associated with burden and readmission outcomes vary with the outcome and the method used 
to measure the outcome. The up-to-date, injury- and outcome-specific AICIs for burden and readmissions, which are similar to the binary index such as the ECM but with fewer conditions, are sufficient for predicting outcomes and does not warrant weighted indices such as the CCI. The AICIs could be further improved by adding more information on comorbidities.

\section{Supplementary Information}

The online version contains supplementary material available at https://doi. org/10.1186/s12913-021-06149-1.

Additional file 1: Table S1. Socio Economic Index for Areas (SEIFA) and country of birth details for the Victorian, NSW and WA study populations. Table S2. Performance of selected model fitting strategies in assessing the effect of comorbidity on selected outcome measures (Victoria). Table S3. Performance of new vs existing comorbidity indices using classification tables (Victoria). Table S4. Conditions included in the injury comorbidity indices for burden, readmissions, CCI and ECM. Table S5. Performance of new comorbidity indices vs existing comorbidity indices in injury sub-groups (Victoria). Table S6. Presence of comorbidity with mean LOS and proportion of patients with readmission outcomes in the NSW and WA study populations ( $>=15$ years). Table S7. Performance of selected model fitting strategies in assessing the effect of comorbidity on selected outcome measures (NSW and WA).

Additional file 2: Appendix A1.1. ROC curves for overnight stay (age $>=15$ years)

Additional file 3: Appendix A1.2. Plots of predicted length of stay (days) vs observed (at least 1 overnight and $\operatorname{LOS}<=30$ ).

Additional file 4: Appendix A1.3. Residual plots for costs.

Additional file 5: Appendix A1.4. ROC curves for all-cause 30-day readmissions (age $>=15$ years).

Additional file 6: Appendix A1.5. ROC curves for non-planned 30-day readmissions (age $>=15$ years).

Additional file 7: Appendix A2. Interaction plots.

Additional file 8: Appendix A3. Model performance details.

Additional file 9: Appendix A4. Internal and external validation details.

Additional file 10: Appendix A5. Using the Australian Injury

Comorbidity Indices.

\section{Abbreviations}

APDC: Admitted Patient Data Collection; AIC: Akaike Information Criterion; AUC: Area Under The Curve; AUD: Australian Dollars; AICl: Australian Injury Comorbidity Index; AlCl-acr: Australian Injury Comorbidity Index for all-cause 30-day readmission; AICl-b: Australian Injury Comorbidity Index for burden; AlCl-cost: Australian Injury Comorbidity Index for costs; AICl-los: Australian Injury Comorbidity Index for LOS; AICl-npr: Australian Injury Comorbidity Index for non-planed 30-day readmissions; AICl-os: Australian Injury Comorbidity Index for overnight stay; AICl-r: Australian Injury Comorbidity Index for readmissions; CHeReL: Centre for Health Record Linkage; CVDL: Centre for Victorian Data Linkage; CCl: Charlson Comorbidity Index; DLB: Data Linkage Branch; ECM: Elixhauser Comorbidity Measure; HMDC: Hospital Morbidity Data Collection; IRR: Incident Rate Ratio; ICD-10AM : International Statistical Classification of Diseases and Related Health Problems, Tenth Revision, Australian Modifications; ICISS : International Statistical Classification of Diseases based Injury Severity Score; IQR: Inter Quartile Range; LOS: Length Of Stay; MACSS: Multipurpose Australian Comorbidity Scoring System; NSW: New South Wales; OR: Odds Ratio; SEIFA: Socio Economic Indexes For Areas; VAED: Victorian Admitted Episodes Dataset; WA: Western Australia

\section{Acknowledgements}

The authors would like to thank the Centre for Victorian Data Linkage, the Centre Health Record Linkage in New South Wales and the Western
Australian Data Linkage Branch and the custodians of data collections for providing linkage services and the data, and Prof. James Harrison at the National Injury Surveillance Unit for providing the survival risk ratios.

\section{Authors' contributions}

The authors are DTF, JB, SN and ZA. DTF and JB conceived the presented idea, developed the theory, analysed and interpreted the data. SN verified the analytical methods and contributed to the interpretation of results while ZA facilitated data acquisition and contributed to the interpretation of findings and formulation of the discussion. JB, SN and ZA supervised DTF in producing the findings of this work. All authors read and approved the final manuscript.

\section{Funding}

This paper was part of a PhD thesis which was supported by the Victorian Injury Surveillance Unit (VISU) funded by the Victorian Government. The PhD student received the Australian Government Research Training Program scholarship stipend during the course of the project. This PhD project was initiated by VISU and therefore funds for purchasing data were supplied VISU. DTF and JB are staff of VISU; however, the role of VISU was solely to be a source of data acquisition funding and supervision support.

\section{Availability of data and materials}

The data that support the findings of this study are available from CVDL, CHeReL and DLB in WA but restrictions apply to the availability of these data, which were used under license for the current study, and so are not publicly available. The authors are not in a position to release the data (in accordance with the agreement with CVDL, CHeReL and DLB in WA).

\section{Ethics approval and consent to participate}

The study was approved by the Monash University Human Research Ethics Committee (Project no: 1256), the New South Wales Population and Health Services Research Ethics Committee (REF: 2017/HRE0601) and the Department of Health WA Human Research Ethics Committee (RGS0000000613). Historical administrative data was used. The research is low risk in that there was no discomfort or risk of harm to the participants. Name, date of birth and other identifiers were removed from the dataset by the data custodians prior to release of the data to the researchers. Due to the magnitude of the dataset, it was impractical to obtain consent. Datasets were extracted by the relevant data linkage units in each state, therefore the authors did not access the raw data; the extracted data were de-identified unit records. Data custodian approvals were obtained from the following data custodians via the data linkage units; Victorian Agency for Health Information, Victorian Cost Data Collection, Registrar of Births, Deaths and Marriages Victoria, NSW Ministry of Health, Department of Health - Government of Western Australia, and the Australian Co-ordinating Registry.

Consent for publication

Not applicable.

\section{Competing interests}

All authors declare no support from any organisation for the submitted work. DTF has received funding support from the Victorian Injury Surveillance Unit (VISU) at Monash University to pay for two datasets and PhD supervision. No other relationships or activities that could appear to have influenced the submitted work.

\section{Author details}

${ }^{1}$ Monash University Accident Research Centre, Monash University, Clayton Campus, 21 Alliance Lane, Clayton 3800, Victoria, Australia. ${ }^{2}$ Victorian Agency for Health Information, 50 Lonsdale Street, Melbourne, Victoria 3000 , Australia.

Received: 21 July 2020 Accepted: 3 February 2021

Published online: 15 February 2021

References

1. Moore L, Stelfox HT, Turgeon AF, Nathens AB, Le Sage N, Émond M, et al. Rates, patterns, and determinants of unplanned readmission after traumatic injury: a multicenter cohort study. Ann Surg. 2014;259(2):374-80. 
2. Morris DS, Rohrbach J, Sundaram LMT, Sonnad S, Sarani B, Pascual J, et al. Early hospital readmission in the trauma population: are the risk factors different? Injury. 2014;45(1):56-60.

3. Librero J, Peiró S, Ordiñana R. Chronic comorbidity and outcomes of hospital care: length of stay, mortality, and readmission at 30 and 365 days. J Clin Epidemiol. 1999:52(3):171-9.

4. Kuwabara K, Matsuda S, Imanaka Y, Fushimi K, Hashimoto H, Ishikawa KB, et al. Injury severity score, resource use, and outcome for trauma patients within a Japanese administrative database. J Trauma Acute Care. 2010;68(2): 463-70.

5. Bochicchio GV, Joshi M, Bochicchio K, Shih D, Meyer W, Scalea TM. Incidence and impact of risk factors in critically ill trauma patients. World $J$ Surg. 2006;30(1):114-8.

6. Farley JF, Harley CR, Devine JW. A comparison of comorbidity measurements to control for confounding in health outcomes studies. Minneapolis: University of Minnesota; 2005.

7. Johansson PA, Farup PG, Bracco A, Vandvik PO. How does comorbidity affect cost of health care in patients with irritable bowel syndrome? A cohort study in general practice. BMC Gastroenterol. 2010;10(1):31.

8. Mackenzie JE, Rivara PF, Jurkovich JG, Nathens BA, Frey PK, Egleston LB, et al. The National Study on costs and outcomes of trauma. J Trauma. 2007; 63(6 Suppl):S54-67.

9. Charlson ME, Pompei P, Ales KL, MacKenzie CR. A new method of classifying prognostic comorbidity in longitudinal studies: development and validation. J Chronic Dis. 1987:40(5):373-83.

10. Elixhauser A, Steiner C, Harris DR, Coffey RM. Comorbidity measures for use with administrative data. Med Care. 1998;36(1):8-27.

11. Quan H, Li B, Couris CM, Fushimi K, Graham P, Hider P, et al. Updating and validating the Charlson comorbidity index and score for risk adjustment in hospital discharge abstracts using data from 6 countries. Am J Epidemiol. 2011;173(6):676-82.

12. van Walraven $C$, Austin $P C$, Jennings $A$, Quan $H$, Forster AJ. A modification of the Elixhauser comorbidity measures into a point system for hospital death using administrative data. Med Care. 2009;47(6):626-33.

13. Yurkovich M, Avina-Zubieta JA, Thomas J, Gorenchtein M, Lacaille D. A systematic review identifies valid comorbidity indices derived from administrative health data. J Clin Epidemiol. 2015;68(1):3-14.

14. Toson B, Harvey LA, Close JC. The ICD-10 Charlson comorbidity index predicted mortality but not resource utilization following hip fracture. J Clin Epidemiol. 2015;68(1):44-51.

15. Australian Consortium for Classification Development. The international statistical classification of diseases and related health problems, tenth revision, australian modification (ICD-10-AM/ACHI/ACS). 10th ed. Darlinghurst: Independent Hospital Pricing Authority; 2012.

16. Centre for Victorian Data Linkage. Data Linkage Results. Personal communication.

17. Master linkage key quality assurance report http://www.cherel.org.au/qua lity-assurance. Accessed 7 Sept 2017.

18. Department of Health. Data linkage - making the right connections. Perth Western Australia: Government of Western Australia - Department of Health; 2016.

19. AlHW:Pointer SC. Trends in hospitalised injury, Australia, 1999-00 to 201011, Injury research and statistics series no 86 cat no INJCAT 162. Canberra: AlHW; 2013.

20. Mitchell RJ, Curtis K, Braithwaite J. Health outcomes and costs for injured young people hospitalised with and without chronic health conditions. Injury. 2017;48(8):1776-83.

21. Bureau of Health Information. Return to acute care following hospitalisation: spotlight on readmissions. Chatswood: NSW Bureau of Health Information; 2015.

22. Fernando DT, Berecki-Gisolf J, Newstead S, Ansari Z. Complications, burden and in-hospital death among hospital treated injury patients in Victoria, Australia: a data linkage study. BMC Public Health. 2019;19(1):798.

23. 6401.0 Consumer Price Index, Australia http://www.abs.gov.au/AUSSTATS/abs@. nsf/DetailsPage/6401.0Mar\%202018?.OpenDocument. Accessed 04 June 2018.

24. Fernando DT, Berecki-Gisolf J, Newstead S, Ansari Z. The Australian injury comorbidity index to predict mortality. Ann Emerg Med. 2020;75(3):339-53.

25. Osler T, Rutledge R, Deis J, Bedrick E. ICISS: an international classification of Disease-9 based injury severity score. J Trauma Acute Care. 1996:41(3):380-8.

26. Australian Institute of Health and Welfare, Henley G, Harrison JE. Injury severity scaling: a comparison of methods for measurement of injury severity, Injury technical paper series no 10. Canberra: AlHW; 2009.
27. Cryer C, Samaranayaka A, Russell D, Davie G, J. L. The epidemiology of serious non-fatal work-related traumatic injury - a demonstration project, Official statistics research series, vol. Vol. 3. Wellington: Injury Prevention Research Unit, University of Otago; 2008.

28. Harrison J, Henley G. Survival risk ratios for Australia; 2008.

29. Quan H, Sundararajan V, Halfon P, Fong A, Burnand B, Luthi J-C, et al. Coding algorithms for defining comorbidities in ICD-9-CM and ICD-10 administrative data. Med Care. 2005;43(11):1130-9.

30. Sundararajan V, Henderson T, Perry C, Muggivan A, Quan H, Ghali WA. New ICD-10 version of the Charlson comorbidity index predicted in-hospital mortality. J Clin Epidemiol. 2004;57(12):1288-94.

31. Akaike H. A new look at the statistical model identification. IEEE Trans Automat Contr. 1974;19(6):716-23.

32. StataCorp. Stata statistical software: release 14. College Station: StataCorp LP; 2015.

33. Vlassoff C. Gender differences in determinants and consequences of health and illness. J Health Popul Nutr. 2007;25(1):47-61.

34. Fernando DT, Berecki-Gisolf J, Newstead S, Ansari Z. Effect of comorbidity on injury outcomes: a review of existing indices. Ann Epidemiol. 2019;36:5-14.

35. Holman CAJ, Preen DB, Baynham NJ, Finn JC, Semmens JB. A multipurpose comorbidity scoring system performed better than the Charlson index. J Clin Epidemiol. 2005:58(10):1006-14.

36. Moore L, Lavoie A, Sage NL, Bergeron É, Émond M, Liberman M, et al. Using information on preexisting conditions to predict mortality from traumatic injury. Ann Emerg Med. 2008;52(4):356-364.e352.

37. Ghali WA, Hall RE, Rosen AK, Ash AS, Moskowitz MA. Searching for an improved clinical comorbidity index for use with ICD-9-CM administrative data. J Clin Epidemiol. 1996;49(3):273-8.

38. Klabunde CN, Potosky AL, Legler JM, Warren JL. Development of a comorbidity index using physician claims data. J Clin Epidemiol. 2000;53(12): 1258-67.

39. Martins $M$, Blais R. Evaluation of comorbidity indices for inpatient mortality prediction models. J Clin Epidemiol. 2006:59(7):665-9.

40. Schneeweiss S, Wang PS, Avorn J, Glynn RJ. Improved comorbidity adjustment for predicting mortality in Medicare populations. Health Serv Res. 2003;38(4):1103-20.

41. Thompson HJ, Rivara FP, Nathens A, Wang J, Jurkovich GJ, Mackenzie EJ. Development and validation of the mortality risk for trauma comorbidity index. Ann Surg. 2010;252(2):370-5.

42. Mitchell RJ, Cameron CM, McClure R. Higher mortality risk among injured individuals in a population-based matched cohort study. BMC Public Health. 2017;17:150.

\section{Publisher's Note}

Springer Nature remains neutral with regard to jurisdictional claims in published maps and institutional affiliations.

Ready to submit your research? Choose BMC and benefit from

- fast, convenient online submission

- thorough peer review by experienced researchers in your field

- rapid publication on acceptance

- support for research data, including large and complex data types

- gold Open Access which fosters wider collaboration and increased citations

- maximum visibility for your research: over $100 \mathrm{M}$ website views per year

At $\mathrm{BMC}$, research is always in progress.

Learn more biomedcentral.com/submission 